\title{
PRESENÇA DE OVOS DE TOXOCARA SPP EM PRAÇAS PÚBLICAS DA CIDADE DE UBERLÂNDIA, MINAS GERAIS, BRASIL.
}

\author{
Julia Maria COSTA-CRUZ, Raquel So uza NUNES \& Abadia Gilda BUSO
}

\begin{abstract}
RESUMO
O objetivo deste trabalho foi verificar a presença de ovos de Toxocara spp nos solos de praças públicas da cidade de Uberlân dia, região do Triângulo Mineiro, no periodo de outubro de 1991 a janeiro de 1992. A cidade possui 89 praças distribuídas $\mathrm{em} 39$ bairros. Para que se tivesse um perfil da ocorrência do parasita, foram colhidas amostras de terra e areia (quando existente) de uma praça, detcrminada por sorteio, por bairro. As amostras de solo homogeneizadas de 5 pontos distintos das 39 praças foram colhidas em frascos plásticos e analisadas empregando-se os métodos de flutuação em solução saturada de cloreto de sódio e de solução saturada de sulfato de magnésio contendo $5 \%$ de iodeto de potássio. Para cada amostra foram realizados os dois mćtodos em duplicata. Os resultados mostraram estarem contaminados os solos de 9 praças $(23,07 \%)$, das quais 6 localizavam-se próximas ao centro da cidade. A realização de mais de um método laboratorial permitiu a identificação do agente em maior porcentagem de locais.
\end{abstract}

UNITERMOS: Toxocara spp; Contaminação do solo.

\section{INTRODUÇño}

O solo contaminado por ovos de Toxocara spp pode ser indicativo da possibilidade de ocorrência de infecção humana em uma localidade.

Os Toxocara canis c o Toxocara cati são as principais espécies que parasitam respectivamente o cão e o gato, suas larvas atingem eventualmente o homem como agentes da Larva Migrans Visceral com quadro clínico bastante variável e inespecílico. Alguns pacientes apresentam-se assintomáticos enquanto outros apresentam quadro mais grave caracterizado por febre, hipereosinofilia, hepatomegalia, manifestações oculares, pulmonares ou cardiacas, nefrose e sinais de lesão cerebral podendo ser fatais. A população de risco é constituida pelas crianças entre 2 e 5 anos de idade que têm maior contato com animais parasitados e com solo onde foram depositados excrementos destes.

Estudo sobre a porcentagem de solo contaminado com ovos de Toxocara canis no periodo de 1973 a 1986 foi realizado por $B \wedge R R I G A^{3}$ que destacou 60 publicações de vários países, observando uma variação de 0,3 a $87 \%$ de positividade. Na Espanha também já foram descritas contaminações de solos de parques públicos ${ }^{2}$ e de áreas urbana e rural ?

A ocorrência de formas evolutivas de Toxocara spp, no Brasil, tem sido estudada em cães, solo e, por métodos soroepidemiológicos, no homem ${ }^{4,5,6,8,10}$.

O objetivo deste trabalho foi verificar a presença de

Laboratório de Parasitologia do Centro de Ciências Biomédicas da Universidade Federal de Uberlândia, Minas Gerais, Brasil.

Endereço para correspondência: Dra. Julia Maria Costa-Cruz. Disciplina de Parasitologia da Universidade Federal de Uberlândia. Av. Pará 1720.

CTP 38400-902. Uberlândia, Minas Gerais, Brasil. Fax: 0342328620. 
ovos de Toxocara spp nos solos de praças públicas da cidade de Uberlândia.

\section{MATERIAL E METODOS}

A cidade de Uberlândia, região do Triângulo Mineiro, Minas Gerais, conta com 89 praças distribuidas em 39 bairros ${ }^{9}$. Foram selecionadas 39 praças, por sorteio, sendo cada uma representante de um bairro, formando assim uma amostra estratificada.

Foi colhida uma amostra de $100 \mathrm{~g}$ do solo de cada praça, no periodo de outubro de 1991 a janeiro de 1992. Cada amostra constituía-se de um homogeneizado de terra e areia (quando existente) de 5 pontos diferentes da praça, obtendo-se desta forma um mapeamento do local. As amostras colhidas de solo úmido, de uma profundidade de aproximadamente $5 \mathrm{~cm}$, foram armazenadas em recipientes de plástico e conservadas a 4 graus Celsius durante, no máximo, 72 horas.

Para cada amostra foram confeccionadas duas lâminas utilizando solução saturada de cloreto de sódio (Método de Willis) ${ }^{12}$ e outras duas utilizando solução saturada de sulfato de magnésio, contendo $5 \%$ de iodeto de potássio "que foram analisadas em microscópio óptico com objetiva de 10 e 40 aumentos, no Laboratório de Parasitologia da Universidade Federal de Uberlândia por dois observadores, sendo que cada um examinou uma lâmina de cada método. $O$ resultado foi considerado positivo quando foram encontrados ovos do parasita.

\section{RESULTADOS}

Das 39 amostras de solos das praças, $9(23,07 \%)$ mostraram-se contaminadas por ovos do Toxocara spp. Em outubro de 1991, foram colhidas amostras de 15 praças, em novembro de 9 , em dezembro de 11 e em janeiro de 4 , sendo respectivamente positivas 2 $(13,33 \%), 3(33,33 \%), 3(27,27 \%)$ e $1(25 \%)$ amostras por mês.

Os bairros cujas praças apresentaram ovos do parasita foram: Copacabana, Lidice, Rezende, Santa Rosa, Satélite, Tabajaras, Taiaman, Tubalina e Vila Oswaldo, correspondendo respectivamente às seguintes praças: de Lourdes, Henckmar Borges, Adalberto Rodrigues da Cunha, Américo Rezende, Chico Mendes, Ronaldo A. Pena, Antônio Carlos Carrijo, São Francisco de Paula e Américo Zardo. Verificou-se que 6 desses bairros

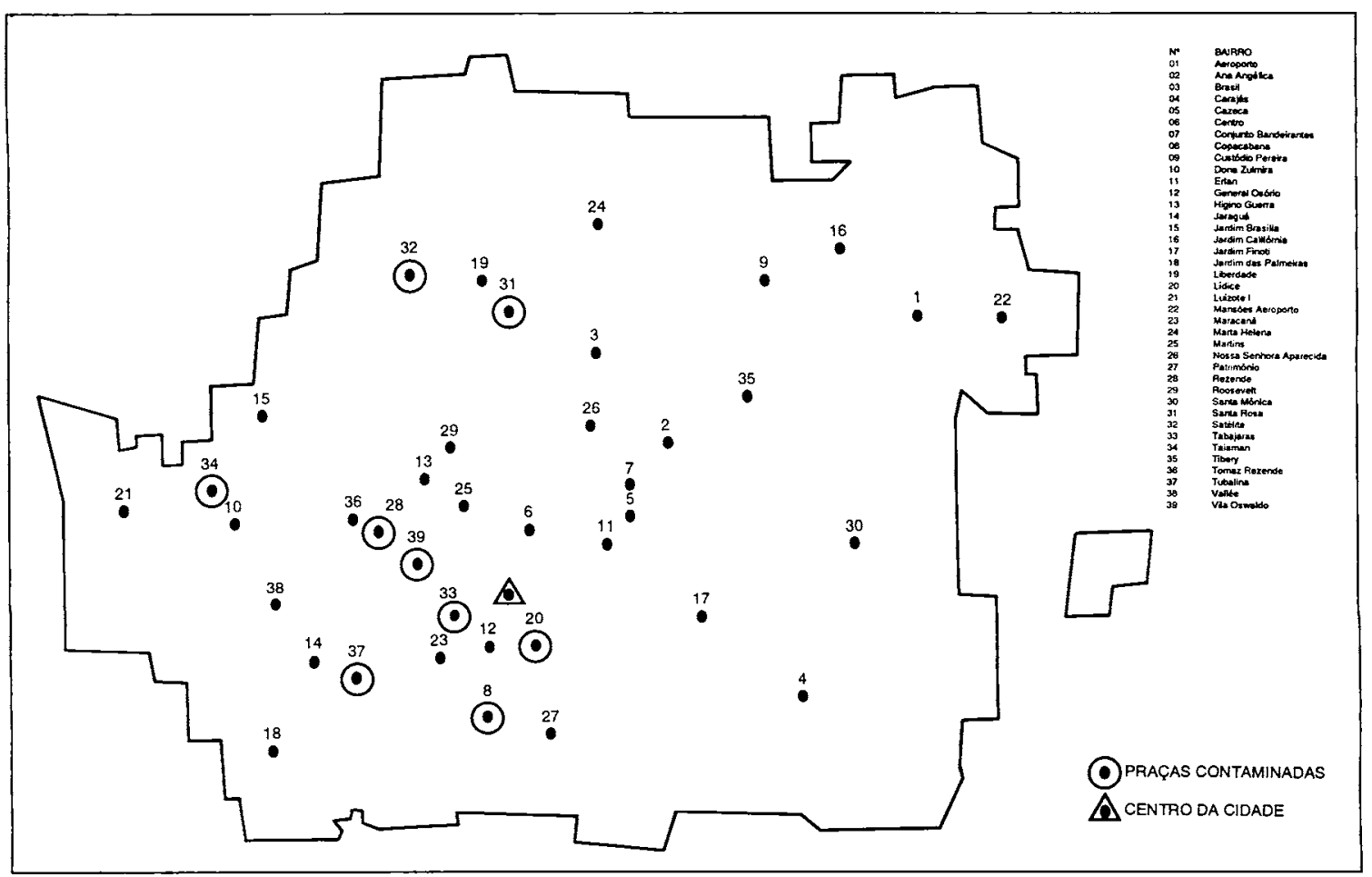

Fig. 1 - Localização das 39 praças públicas da cidade de Uberlândia, destacando as 9 contaminadas por ovos de Toxocara spp no período de outubro de 1991 a janciro de 1992. 
(66,66\%) estão localizados próximos ao centro da cidade (Figura 1).

O parasita foi detectado pelo método de Willis em 5 praças e pelo método do sulfato de magnésio contendo $5 \%$ de iodeto de potássio em outras 4 .

\section{DISCUSSÃO}

Os resultados obtidos demonstram pioneiramente a presença de ovos de Toxocara spp em praças públicas da cidade de Uberlândia, Minas Gerais.

Foi possivel conhecer o perfil da distribuição da contaminação do solo em Uberlândia uma vez que a coleta abrangeu uma praça de cada bairro da cidade e das 9 positivas, 6 estavam localizadas próximas ao centro da cidade. Nenhum outro ovo de helminto ou cisto de protozoário foi observado.

Com referência aos trabalhos publicados no Brasil, o grau de contaminação encontrado $(23,07 \%)$ foi equivalente aos achados de ALCÂNTARA et al. ' na Bahia (24,8\%); superior aos de PEREIRA DA SILVA (17\%) no Rio de Janeiro ${ }^{10} \mathrm{e}$ inferior aos de FERREIRA et al. ${ }^{8}$ no Rio de Janeiro $(41,6 \%)$ e CHIEFFI \& MÜLLER ${ }^{4,5}$ em Londrina, com respectivamente $60 \mathrm{e}$ $100 \%$ de confirmação diagnóstica em solos pesquisados.

No Brasil, a diversidade de métodos utilizados dificulta a comparação dos resultados. A opção pelos dois métodos utilizados neste trabalho tem como fundamento a publicação de QUINN et al. ${ }^{11}$ que, comparando 7 métodos, verificaram ser o de sulfato de magnésio, contendo $5 \%$ de iodeto de potássio, o de maior sensibilidade.

A presença de ovos de Toxocara spp em solos de praças públicas indica a possibilidade de ocorrência de infeç̧ão humana em Uberlândia.

\section{SUMMARY}

Presence of Toxocara spp eggs in public squares of Uberlândia city, Minas Gerais, Brazil.

The objective of this study was to verify the presence of Toxocara spp eggs in the public squares ground in Uberlândia, region of Triângulo Mineiro, from October 1991 to January 1992. The city has 89 squares distributed in 39 boroughs. In order to have an outline of the parasite's occurrence, ground and sand (when existent) samples were collected from one square per boroughors. The squares were determined by draw. The homogenized ground samples of distinct points of the 39 squares, were colected in plastic flasks and analysed by the methods of nuctuation in sodium chloride saturated solution and magnesium sulphate saturated solution which contained $5 \%$ of potassium iodate. Both methods were executed twice for each sample. The results showed that the ground of 9 squares $(23.07 \%)$ was contaminated, 6 of which were located near downtown. The execution of more than one laboratorial method permited the agent's identification in a grater percentage of squares.

\section{REFERENCIAS BIBLIOGRÁFICAS}

1. ALCÂNTARA, N.; BAVIA, E.; Silvĩo, R. M. \& CARVALHO, E. - Environmental contamination by Toxocara sp eggs in public areas of Salvador, Bahia state, Brazil. Rev. Soc. bras. Med. trop., 22: 187-190, 1989.

2. ANGUlo-madero, R.; AGUIla DE la pUENTE, C. \& GUILLENLLERA, J. L. - Contaminación de sueios de parques publicos por Toxocara canis. Rev. ibér. Parasit., 47 (supl.): 165 $171,1987$.

3. BARRIGA, O. O. - A critical look at the importance, prevalence and control of toxocariasis and the possibilities of immunological control. Vet. Parasit., 29: 195-234, 1988.

4. CIIEFFI, P. P. \& MOLLER, E. E. - Prevalência de parasitismo por Toxocara canis em cães e presença de ovos de Toxocara sp no solo de localidades públicas da zona urbana do municipio de Londrina, Estado do Paraná, Brasil. Rev. Saúde públ. (S. Paulo), 10: 367372, 1976.

5. CHIEFF, P. P. \& MULLER, E. E. - Estudo de variação na contaminação do solo por ovas de Toxocara sp (Nematode, Ascaroidea), na zona urbana do municipio de Londrina, Estado do Paraná, Brasil. Rev. Inst. Adolfo Lutz, 38: 13-16, 1978.

6. CHIEFFI, P. P.; UEDA, M.; CAMARGO, E. D. et al. - Visceral Larva Migrans: a seroepidemiological survey in five municipalities of São Paulo State, Brazil. Rev. Inst. Med. trop. S. Paulo, 32: 204-210, 1990.

7. CONDE GARCIA, L.; MURO ALVAREZ, A. \& SIMON MARTIN, F. - Epidemiological studies on toxocariasis and Visceral Larva Migrans in a zone of westem Spain. Ann. trop. Med. Parasit., 83: $615-620,1989$.

8. FERREIRA, L. F.; OLIVEIRA, E. L. \& CAMLLLO-COURA, L. Sobre a presença de ovos de Toxocara em praças da cidade do Rio de Janeiro. Rev. Soc. bras. Med. trop., 10: 51-54, 1976.

9. GUIA SEI - Serviços - endereços - informaçōes. Uberlândia, 1990/1991. p. $423-468$. 
COSTA-CRUZ, J. M.; NUNES, R. S. \& BUSO, A. G. - Presença de ovos de Toxocara spp em praças públicas da cidade de Uberlândia, Minas Gerais, Brasil. Rev. Inst. Med. trop. S. Paulo, 36(1):39-42, 1994.

10. PEREIRA DA SILVA, J. - Contaminação de praças do município do Rio de Janeiro por ovos de helmintos. Atas Soc. biol. (Rio de J.), 24: $1-2,1984$.

11. QUINN, R.; SMITH, H. V.; BRUCE, R. G. \& GIERDWOOD, R. W. A. - On the incidence of Toxocara and Toxocaris spp ova in the environment. 1. A comparison of flolation procedures for recovering Toxocara spp ova from soil. J. Hyg. (Lond.), 84: $83-89,1980$.

12. WILLIS, HI. H. - A simple levitation method for the detection of hookworm ova. Med. J. Aust., 8: 375-376, 1921.

Recebido para publicação em 12/4/93.

Aceito para publicação em $9 / 9 / 93$. 